\title{
Bortezomib-based chemotherapy to treat refractory angioimmunoblastic T-cell lymphoma: A case report and review of the literature
}

\author{
HUA-PING DU, QIAN-QIAN YANG and YE ZHANG \\ Department of Hematology, Sir Run Run Shaw Hospital, Zhejiang University School of Medicine, \\ Hangzhou, Zhejiang 310016, P.R. China
}

Received December 26, 2014; Accepted January 12, 2016

DOI: $10.3892 / \mathrm{ol} .2016 .4213$

\begin{abstract}
The peripheral T-cell lymphomas (PTCLs) are a heterogeneous group of aggressive neoplasms that account for $<15 \%$ of all non-Hodgkin's lymphoma cases in adults. Angioimmunoblastic T-cell lymphoma (AITL) is a specific subtype of PTCL. The tumor is frequently aggressive and there is currently no general consensus regarding an effective treatment strategy. The present study reports a case in which bortezomib combined with dexamethasone was used to treat refractory AITL. A 63-year-old woman was admitted to Sir Run Run Shaw Hospital, Zhejiang University School of Medicine (Zhejiang, China) on August 17, 2013. The patient had been diagnosed with AITL for 4 months and had experienced a relapse of symptoms for the 4 days prior to admission. The patient demonstrated fever and dyspnea, accompanied by severe edema in the face and lower limbs, which later spread to the right upper limb. The patient was treated with bortezomib plus dexamethasone, which rapidly relieved the symptoms. The patient was subsequently administered an additional 2 cycles of bortezomib-based chemotherapy and survived for an additional 4 months, prior to succumbing to the disease. Only a small number of studies have reported the use of bortezomib in the treatment of T-cell lymphoma. The present study suggested that bortezomib-based treatment may be a reliable, safe and effective alternative for the treatment of relapsed/refractory PTCL. The efficacy of bortezomib as a treatment for PTCL requires additional evaluation in future studies.
\end{abstract}

Correspondence to: Dr Hua-Ping Du, Department of Hematology, Sir Run Run Shaw Hospital, Zhejiang University School of Medicine, 3 East Qing Chun Road, Hangzhou, Zhejiang 310016, P.R. China E-mail: duhuaping@163.com

Key words: lymphoma, bortezomib, superior vena cava syndrome, chemotherapy

\section{Introduction}

Peripheral T-cell lymphomas (PTCL) are a heterogeneous group of aggressive neoplasms that account for $<15 \%$ of all non-Hodgkin's lymphoma (NHL) cases in adults (1). The incidence of PTCL varies geographically, with the highest incidence in regions of Asia $(2,3)$. The incidence in the USA has increased by $7.9 \%$ annually between 1992 and 2005 (4). In total, there are 23 types of PTCL defined by the 2008 World Health Organization classification (5), including extranodal nasal-type natural killer/T-cell lymphoma, enteropathy-associated T-cell lymphoma, subcutaneous panniculitis-like cell lymphoma, angioimmunoblastic T-cell lymphoma (AITL), anaplastic large cell lymphoma (ALCL) and peripheral T-cell lymphomas not otherwise specified (PTCL-NOS). There are clear variations in incidence and survival rates in the subtypes according to age, gender, ethnicity and geographical distribution $(2,6)$. A diagnosis of PTCL is based on the results of a tissue biopsy, usually a lymph node biopsy. The disease stage and prognosis of the patient at diagnosis are determined by the International Prognostic Index (IPI) score, similarly to other NHLs $(7,8)$. PTCL is frequently aggressive and there is currently no general consensus regarding an effective treatment strategy; therefore, regimens that treat aggressive B-cell NHL are commonly used (4). Among all the subtypes anaplastic lymphoma kinase positive ALCL may have the best survival rate, while patients with other PTCLs have poor outcomes (1). The tendency for poor survival rates in PTCL suggests that the regimens used in patients with aggressive B-cell lymphomas may not be equally efficacious in T-cell lymphomas. Therefore, patients are encouraged to attend clinical trials whenever available (9).

PTCL-NOS is the most common subtype of PTCL, accounting for $\sim 26 \%$ of T-cell lymphoma cases worldwide, and AITL is the second most common subtype accounting for $\sim 19 \%$ (6). The majority of patients with AITL have a median age of 59-64 years without any gender predilection (10). Patients typically present with generalized lymphadenopathy, fever, night sweats and weight loss (10). AITL may manifest following the administration of drugs, particularly antibiotics, or a viral infection, and may occasionally be associated with various bacterial or fungal infections, possibly reflecting the 
consequences of immune deregulation in AITL patients (9). The course of AITL is variable, and the overall prognosis is poor; however, there is occasional spontaneous remissions in a few cases (9). The best treatment for AITL is unknown. Anthracycline-based chemotherapy, administered following induction therapy, may achieve a $46 \%$ complete remission rate; however, intensive combination chemotherapy, including CHOP (cyclophosphamide, doxorubicin, vincristine and prednisone), hyper-CVAD (cyclophosphamide, doxorubicin, vincristine and dexamethasone) and $\mathrm{mBACOD}$ (methotrexate, bleomycin, doxorubicin, cyclophosphamide, vincristine and dexamethasone) did not improve complete remission rates when used with various chemotherapeutics during randomized studies, including stem cell transplantation (10). Univariate analysis demonstrated that men, mediastinal lymphadenopathy and anemia are poor prognostic factors for the overall survival rate of patients (10). No therapy has increased the long-term survival rate to $>30 \%$ (6).

The present study reports a case of refractory AITL, which was treated with bortezomib combined with dexamethasone.

\section{Case report}

A 63-year-old woman was admitted to the Sir Run Run Shaw Hospital, Zhejiang University School of Medicine (Zhejiang, China) on August 17, 2013. The patient had been diagnosed with AITL for 4 months and had experienced a relapse of symptoms for the preceding 4 days prior to admission.

A total of 4 months prior to admission, the patient presented with enlarged bilateral cervical, axillary and inguinal lymph nodes, which exhibited moderate hardness and limited motion. Computed tomography (CT; SOMATOM Definition AS and SOMATOM Sensation 16; Siemens AG, Munich, Germany) revealed hepatosplenomegaly and lymphadenopathy at the mediastinal, retroperitoneal and mesenteric regions.

Pathological assessment of a left lymph node biopsy led to a diagnosis of AITL, with the following immunohistochemical results (all antibodies were mouse anti-human monoclonal, unless otherwise stated): Negative staining for cluster of differentiation (CD)20 (catalog no., M0755; dilution, 1:1,500; Dako, Glostrup, Denmark) and CD8 (catalog no., NCL-LCD8-295; dilution, 1:50; Leica Biosystems Nussloch GmbH, Nussloch, Germany); positivity for CD3 (catalog no., A0452; dilution, 1:400; Dako), Ki-67 (80\%; catalog no., M7240; dilution, 1:1,000; Dako), B-cell lymphoma 6 (catalog no., NCL-L-BCL-6-564; dilution, 1:400; Leica Biosystems Nussloch $\mathrm{GmbH}$ ), CD4 (catalog no., M7310; dilution, 1:50; Dako), programmed cell death protein 1 (catalog no., ZM-0381; dilution, 1:1,500; Beijing Zhongshan Biological Technology Co., Ltd., Beijing, China) and CD5 (catalog no., M3641; dilution, 1:50; Dako); partial positivity for CD7 (catalog no., NCL-L-CD7-580; dilution, 1:400; Leica Biosystems Nussloch $\mathrm{GmbH}$ ) and chemokine (C-X-C motif) ligand 13 (goat polyclonal; catalog no., ZG-0601; dilution, 1:100; Beijing Zhongshan Biological Technology Co., Ltd.); focal staining for CD10 (catalog no., M7308; dilution, 1:50; Dako); positivity for CD21 (catalog no., M0784; dilution, 1:1,000; Dako) indicating dendritic cell hyperplasia; and Epstein-Barr virus positivity in scattered cells. T-cell receptor rearrangement was positive. Furthermore, bone marrow biopsy revealed abnormal T-cell infiltration. The patient was diagnosed with stage IV AITL and B-group, and experienced night sweats and weight loss (6). The patient demonstrated an IPI (7) score of 2, due to the presence of stage IV disease and being $>60$ years of age.

The patient was treated withcyclophosphamide $(1,000 \mathrm{mg} / \mathrm{dl}$; Jiangsu Hengrui Medicine Co., Ltd., Lianyungang, China), doxorubicin (70 mg/dl; Shenzhen Main Luck Pharmaceuticals Inc., Shenzhen, China), vincristine (2 mg/dl; Zhejiang Hisun Chemical Co., Ltd., Taizhou, China) and prednisone (30 mg/dl; Zhejiang Xianju Pharmaceutical Co., Ltd., Hangzhou, China) (CHOP) plus L-asparagine (L-ASP; 3,600 IU day 1; Jiangsu Hengrui Medicine Co., Ltd.) chemotherapy on May 8, 2013. However, L-ASP treatment was subsequently ceased due to a severe skin rash. Subsequently, the patient was administered 2 cycles of CHOP chemotherapy. The enlarged lymph nodes were observed to shrink following the first cycle of chemotherapy, but became larger again and were accompanied by fever prior to the next cycle of chemotherapy; therefore, the therapy was changed to a gemcitabine $(1,400 \mathrm{mg} / \mathrm{dl}$; Faulding Pharmaceuticals, Ltd., Adelaide, Australia), dexamethasone (40 mg/dl on days 1-4; Jiangsu Hansoh Pharmaceutical, Co., Ltd., Lianyungang, China) and platinum (Jiangsu Hansoh Pharmaceutical, Co., Ltd.) regimen. Subsequently, the lymph nodes were observed to have decreased in size.

Between August 13-17, 2013, the patient exhibited a fever $\left(39^{\circ} \mathrm{C}\right)$ and dyspnea. Physical examination revealed severe edema in the face and lower limbs. The symptoms were not relieved following 1 week of antibiotic treatment with imipenem and caspofungin, and diuretic treatment $(20 \mathrm{mg}$ intravenous furosemide; Shanghai Harvest Pharmaceutical, Co., Ltd., Shanghai, China). The patient was weak and was confined to bed rest. The facial edema became more severe and spread to the right upper limb. The patient additionally exhibited abdominal distention. Chest and abdominal CT revealed a small amount of pleural, mediastinal and abdominal effusion. Mediastinal, supraclavicular and peripancreatic lymph nodes were enlarged. The patient's condition became increasingly worse, and the patient was considered to exhibit superior vena cava (SVC) syndrome, which is one complication of lymphoma. On August 21, 2013, the patient was administered bortezomib plus dexamethasone [2.4 mg bortezomib on days 1 and 4 (Xi'an Janssen Pharmaceutical., Co., Ltd., Xi'an); and $20 \mathrm{mg}$ dexamethasone on days 1-4 (Tianjin Kingyork Group, Tianjin, China)]. Following chemotherapy, the patient no longer exhibited a fever and the antibiotic treatment was ceased. The symptoms improved and 1 week later the patient was discharged from hospital. On September 9, 2013, the patient was followed-up and demonstrated no fever or edema, with no requirement for bed rest. CT indicated that the pleural effusion had decreased and that the peripancreatic, retroperitoneal and mesenteric lymph nodes had reduced in size. The patient was administered another regimen of bortezomib plus dexamethasone on September 11, 2013. However, 1 month later the patient exhibited a red itching rash on the neck and face. The patient was administered bortezomib and a hyper-CVAD A regimen (2.4 mg bortezomib on days 1 and 4; $400 \mathrm{mg}$ cyclophosphamide twice a day, on days 1-3; $60 \mathrm{mg}$ Adriamycin on day $4 ; 2 \mathrm{mg}$ vincristine on days 4 and 11; and $40 \mathrm{mg}$ dexamethasone on days 1-4 and 11-14). Due to bone marrow suppression, the patient developed a severe infection 
following chemotherapy. Therefore, chemotherapy was ceased and the patient succumbed to the disease on January 6, 2014. Written informed consent was obtained from the patient's family for the publication of the present study.

\section{Discussion}

PTCLs are a heterogeneous group of aggressive neoplasms and patient survival depends, at least partially, upon the subtype identified. In general, survival time is measured in months without treatment. The International Peripheral T-cell Lymphoma Project reported 5-year overall survival rates of $32 \%$ and 5-year relapse-free survival of only $20 \%$ in subtypes of PTCL not otherwise specified (PTCL-NOS), while the survival rates were 33 and $18 \%$ in AITL, respectively $(11,12)$. It is clear that patients with T-cell lymphoma possess inferior rates of response to chemotherapy, and demonstrate poorer progression-free survival (PFS) and overall survival compared with patients with B-cell lymphoma (13). There is no general consensus regarding the preferred chemotherapeutic treatment for PTCL, and patients should be encouraged to participate in clinical trials whenever possible (14).

Bortezomib, a proteasome inhibitor, is a novel agent approved by the US Food and Drug Administration (FDA) for the treatment of multiple myeloma and relapsed or refractory mantle cell lymphoma (15-17). However, only a few studies have reported the use of bortezomib to treat T-cell lymphoma (Table I).

A study by Liu et al (18) described the case of a 76-year-old female patient with AITL, who did not respond to conventional chemotherapy, but responded to bortezomib-based treatment. The treatment regimen was PAD, comprising $1.3 \mathrm{mg} / \mathrm{m}^{2}$ bortezomib, $2 \mathrm{mg} / \mathrm{m}^{2}$ mitoxantrone and $40 \mathrm{mg}$ dexamethasone on days $1,4,8$ and 11 for 5 cycles of 28 days each (18). The patient demonstrated persistent clinical improvement, weight gain, disappearance of lymphadenopathy, improvement of performance status and no systemic symptoms of fever, night sweats or weight loss at the 18-month follow-up visit (18). Hourigan et al (19) also used bortezomib accompanied by an EPOCH regimen (etoposide, vincristine, doxorubicin, cyclophosphamide and prednisone) as a salvage therapy for the treatment of a 48-year-old man who was diagnosed with refractory acute adult T-cell leukemia/lymphoma (ATL). Bortezomib $\left(1 \mathrm{mg} / \mathrm{m}^{2}\right)$ was administered on days $1,4,8$ and 11 of each cycle (19). Following a single cycle of V-EPOCH (bortezomib plus EPOCH) (20), the lymphadenopathy resolved and the patient's lactate dehydrogenase levels became normalized (19). Following 4 cycles of V-EPOCH, peripheral blood flow cytometry and a bone marrow examination demonstrated no evidence of residual leukemia (19). Subsequent to 5 cycles of V-EPOCH, the patient received a consolidative non-myeloablative haploidentical bone marrow transplant and continued to demonstrate a complete response (CR) 36 months after the completion of treatment (19).

There have been a number of prospective phase II trials of bortezomib in patients exhibiting relapsed or refractory PTCL. Kim et al (21) performed a phase II study on 46 patients with stage III/IV PTCL, to investigate the efficacy of bortezomib in combination with $\mathrm{CHOP}$ as a first-line treatment. Patients were administered bortezomib on days 1 and 8 
at a dose of $1.6 \mathrm{mg} / \mathrm{m}$ (22), in addition to CHOP every 3 weeks for a total of 6 cycles (21). Overall, three subtypes of PTCL (PTCL-NOS, AITL and anaplastic lymphoma kinase-negative anaplastic large-cell lymphoma) demonstrated an $87 \%$ overall response rate (ORR) and a $73 \% \mathrm{CR}$ rate $(21)$. However, the treatment efficacy for extranodal natural killer (NK)/T-cell lymphoma, nasal type was poor with a CR rate of only $30 \%$ (3/10) (21). In total, 30 patients achieved a CR $(65 \%)$ and the ORR was $76 \%$ (12). The 3-year overall survival and progression-free survival rates were 47 and $35 \%$, respectively, due to frequent relapse following remission. However, bortezomib plus CHOP appears to be superior to treatment with $\mathrm{CHOP}$ alone or CHOP-like regimens (21). Lee et al (22) also investigated bortezomib in combination with $\mathrm{CHOP}$ as a first-line therapy for advanced, aggressive T-cell lymphoma. A total of 13 patients received 55 cycles of treatment. The overall CR rate in all patients was $61.5 \%$ (22). Zinzani et al (23) used bortezomib as a single agent to treat cutaneous T-cell lymphoma, at a dose of $1.3 \mathrm{mg} / \mathrm{m}^{2}$, intravenously on days $1,4,8$ and 11 , every 21 days for a total of 6 cycles. The ORR was $67 \%$, with $2(17 \%)$ and $6(50 \%)$ patients achieving a CR and a partial response (PR), respectively (23). All responses were enduring, lasting for 7-14 months (23). In all the aforementioned studies, bortezomib was well-tolerated. The most common toxicities were peripheral sensory neuropathy, neutropenia and thrombocytopenia.

The primary mechanism underlying the anticancer activity of bortezomib is via the activation of nuclear factor- $\kappa \mathrm{B}(\mathrm{NF}-\kappa \mathrm{B})$ and inhibition of the degradation of inhibitory- $\kappa \mathrm{B}$, which leads to the suppression of the NF- $\mathrm{BB}$ signaling pathway, followed by downregulation of anti-apoptotic target genes (24). Another important anticancer mechanism occurs via upregulation of NOXA, which is a pro-apoptotic protein that may interact with the anti-apoptotic proteins of the B-cell lymphoma 2 (Bcl-2) subfamily, leading to the apoptotic death of malignant cells. Bortezomib-induced apoptosis has been demonstrated in $\mathrm{T}$ and NK lymphoma cells, implying that bortezomib may have a significant therapeutic role in the treatment of T-cell lymphoma (25-27).

The patient in the present study was diagnosed with stage IV refractory PTCL, and appeared to exhibit primary drug resistance. Classical chemotherapy was not effective. The patient subsequently developed severe edema of the face and lower limbs. CT scans revealed enlarged lymph nodes in the mediastinal and supraclavicular regions. It was considered that the patient may by exhibiting SVC syndrome, despite negative indications on the CT scan. SVC syndrome is a condition caused by the obstruction of blood flow through the $\operatorname{SVC}(28,29)$. Obstruction may be caused by invasion or external compression of the SVC by an adjacent pathological process involving the right lung, lymph nodes or other mediastinal structures, or by thrombosis. Dyspnea is the most common symptom, and patients also frequently exhibit facial swelling or head fullness, arm swelling, a cough, chest pain or dysphagia. Patients with cerebral edema may experience headaches, confusion or possibly become comatose. Upon physical examination, the most common findings are facial edema, and distension of the veins in the neck and on the chest wall. The most frequent cause of SVC syndrome is intrathoracic malignancy, which is responsible for $60-85 \%$ of cases. Among all malignancies, lung cancer and NHL are responsible for $\sim 95 \%$ of cases of SVC syndrome $(28,29)$. Non-malignant conditions account for $15-40 \%$ of SVC obstructions in contemporary retrospective series. Among these cases, SVC thrombosis cases, which are associated with the presence of intravascular devices, including central venous catheters and cardiac pacemaker leads, have increased rapidly $(29,30)$. The goal of management for SVC syndrome is to alleviate symptoms and treat the underlying disease. For patients with malignancy, chemotherapy is the initial choice of treatment. The edema symptoms of the present patient were relieved soon after chemotherapy, indicating the effectiveness of the treatment.

For the present patient, who exhibited refractory and aggressive T-cell lymphoma, bortezomib with dexamethasone achieved rapid and favorable effects. The symptoms caused by SVC syndrome were completely relieved and the lymph nodes reduced in size. The results of the present study suggest that bortezomib-based treatment may be a reliable, safe and effective alternative for the treatment of relapsed/refractory PTCL. However, the efficacy of bortezomib should be additionally evaluated in larger controlled clinical trials, with an extended follow-up period. Further investigation is required to observed whether bortezomib is more favorable for the treatment of AITL than other drugs.

\section{References}

1. Armitage JO: The aggressive peripheral T-cell lymphomas: 2013 Am J Hematol 88: 910-918, 2013.

2. Abouyabis AN, Shenoy PJ, Lechowicz MJ and Flowers CR: Incidence and outcomes of the peripheral T-cell lymphoma subtypes in the United States. Leuk Lymphoma 49: 2099-2107, 2008.

3. Anderson JR, Armitage JO and Weisenburger DD: Epidemiology of the non-Hodgkin's lymphomas: Distributions of the major subtypes differ by geographic locations. Non-Hodgkin's Lymphoma Classification Project. Ann Oncol 9: 717-720, 1998.

4. Phillips AA, Owens C, Lee S and Bhagat G: An update on the management of peripheral T-cell lymphoma and emerging treatment options. J Blood Med 2: 119-129, 2011.

5. Swerdlow SH, Campo E, Harris NL, Jaffe ES, Pileri SA, Stein H, Thiele J and Vardiman JW (eds): WHO Classification of Tumours of Haematopoietic and Lymphoid Tissues. Vol 2. 4th edition. IARC, Lyon, 2008

6. Vose J, Armitage J and Weisenburger D; International T-Cell Lymphoma Project: International peripheral T-cell and natural killer/T-cell lymphoma study: Pathology findings and clinical outcomes. J Clin Oncol 26: 4124-4130, 2008.

7. Lister TA, Crowther D, Sutcliffe SB, Glatstein E, Canellos GP, Young RC, Rosenberg SA, Coltman CA and Tubiana M: Report of a committee convened to discuss the evaluation and staging of patients with Hodgkin's disease: Cotswolds meeting. J Clin Oncol 7: 1630-1636, 1989 .

8. The International Non-Hodgkin's Lymphoma Prognostic Factors Project: A predictive model for aggressive non-Hodgkin's lymphoma. N Engl J Med 329: 987-994, 1993.

9. de Leval L, Gisselbrecht C and Gaulard P: Advances in the understanding and management of angioimmunoblastic T-cell lymphoma. Br J Haematol 148: 673-689, 2010.

10. Mourad N, Mounier N, Brière J, Raffoux E, Delmer A, Feller A, Meijer CJ, Emile JF, Bouabdallah R, Bosly A, et al: Clinical, biologic, and pathologic features in 157 patients with angioimmunoblastic T-cell lymphoma treated within the Groupe d'Etude des Lymphomes de l'Adulte (GELA) trials. Blood 111: 4463-4470, 2008.

11. Weisenburger DD, Savage KJ, Harris NL, Gascoyne RD, Jaffe ES, MacLennan KA, Rüdiger T, Pileri S, Nakamura S, Nathwani B, et al; International Peripheral T-cell Lymphoma Project: Peripheral T-cell lymphoma, not otherwise specified: A report of 340 cases from the International Peripheral T-cell Lymphoma Project. Blood 117: 3402-3408, 2011. 
12. Federico M, Rudiger T, Bellei M, Nathwani BN, Luminari S, Coiffier B, Harris NL, Jaffe ES, Pileri SA, Savage KJ, et al: Clinicopathologic characteristics of angioimmunoblastic T-cell lymphoma: Analysis of the international peripheral T-cell lymphoma project. J Clin Oncol 31: 240-246, 2013.

13. Coiffier B, Brousse N, Peuchmaur M, Berger F, Gisselbrecht C, Bryon PA and Diebold J: Peripheral T-cell lymphomas have a worse prognosis than B-cell lymphomas: A prospective study of 361 immunophenotyped patients treated with the LNH-84 regimen. The GELA (Groupe d'Etude des Lymphomes Agressives). Ann Oncol 1: 45-50, 1990.

14. Lunning MA: Treatment of peripheral T-cell lymphoma: Many shades of gray. Oncology (Williston Park) 29: 545-550, 2015

15. Kyle RA and Rajkumar SV: Multiple myeloma. Blood 111: 2962-2972, 2008.

16. Richardson PG, Barlogie B, Berenson J, Singhal S, Jagannath S, Irwin D, Rajkumar SV, Srkalovic G, Alsina M, Alexanian R, et al: A phase 2 study of bortezomib in relapsed, refractory myeloma. N Engl J Med 348: 2609-2617, 2003.

17. Goy A, Bernstein SH, Kahl BS, Djulbegovic B, Robertson MJ, de Vos S, Epner E, Krishnan A, Leonard JP, Lonial S, et al: Bortezomib in patients with relapsed or refractory mantle cell lymphoma: Updated time-to-event analyses of the multicenter phase 2 PINNACLE study. Ann Oncol 20: 520-525, 2009.

18. Liu S, Yuan Z, Zhang C, Fu W and Hou J: Bortezomib-based treatment for relapsed and refractory angioimmunoblastic T-cell lymphoma: Case report and literature review. Oncol Lett 4 262-264, 2012

19. Hourigan CS, Forde PM, Ambinder RF and Gladstone DE: Bortezomib salvage therapy in refractory acute adult T-cell leukemia/lymphoma. Leuk Lymphoma 54: 2563-2564, 2013.

20. Wilson WH, Bryant G, Bates S, Fojo A, Wittes RE, Steinberg SM, Kohler DR, Jaffe ES, Herdt J, Cheson BD, et al: EPOCH chemotherapy: Toxicity and efficacy in relapsed and refractory non-Hodgkin's lymphoma. J Clin Oncol 11: 1573-1582, 1993

21. Kim SJ, Yoon DH, Kang HJ, Kim JS, Park SK, Kim HJ, Lee J, Ryoo BY, Ko YH, Huh J, et al; Consortium for Improving Survival of Lymphoma (CISL) investigators: Bortezomib in combination with CHOP as first-line treatment for patients with stage III/IV peripheral T-cell lymphomas: A multicentre, single-arm, phase 2 trial. Eur J Cancer 48: 3223-3231, 2012.
22. Lee J, Suh C, Kang HJ, Ryoo BY, Huh J, Ko YH, Eom HS, Kim K, Park K and Kim WS: Phase I study of proteasome inhibitor bortezomib plus CHOP in patients with advanced, aggressive T-cell or NK/T-cell lymphoma. Ann Oncol 19: 2079-2083, 2008.

23. Zinzani PL, Musuraca G, Tani M, Stefoni V, Marchi E, Fina M, Pellegrini C, Alinari L, Derenzini E, de Vivo A, et al: Phase II trial of proteasome inhibitor bortezomib in patients with relapsed or refractory cutaneous T-cell lymphoma. J Clin Oncol 25: 4293-4297, 2007.

24. Chen D, Frezza M, Schmitt S, Kanwar J and Dou QP: Bortezomib as the first proteasome inhibitor anticancer drug: Current status and future perspectives. Curr Cancer Drug Targets 11: 239-253, 2011.

25. Ri M, Iida S, Ishida T, Ito A, Yano H, Inagaki A, Ding J, Kusumoto S, Komatsu H, Utsunomiya A and Ueda R: Bortezomib-induced apoptosis in mature T-cell lymphoma cells partially depends on upregulation of Noxa and functional repression of Mcl-1. Cancer Sci 100: 341-348, 2009.

26. Shen L, Au WY, Guo T, Wong KY, Wong ML, Tsuchiyama J, Yuen PW, Kwong YL, Liang RH and Srivastava G: Proteasome inhibitor bortezomib-induced apoptosis in natural killer (NK)-cell leukemia and lymphoma: An in vitro and in vivo preclinical evaluation. Blood 110: 469-470, 2007.

27. Iwata S, Yano S, Ito Y, Ushijima Y, Gotoh K, Kawada J, Fujiwara S, Sugimoto K, Isobe $\mathrm{Y}$, Nishiyama $\mathrm{Y}$ and Kimura $\mathrm{H}$ : Bortezomib induces apoptosis in T lymphoma cells and natural killer lymphoma cells independent of Epstein-Barr virus infection. Int J Cancer 129: 2263-2273, 2011.

28. Rice TW, Rodriguez RM and Light RW: The superior vena cava syndrome: Clinical characteristics and evolving etiology. Medicine (Baltimore) 85: 37-42, 2006.

29. Yellin A, Rosen A, Reichert N and Lieberman Y: Superior vena cava syndrome. The myth - the facts. Am Rev Respir Dis 141: 1114-1118, 1990

30. Chee CE, Bjarnason $\mathrm{H}$ and Prasad A: Superior vena cava syndrome: An increasingly frequent complication of cardiac procedures. Nat Clin Pract Cardiovasc Med 4: 226-230, 2007. 